\title{
A Computer Based Support to Guided Note Taking: A Preliminary Study on University Students with Dyslexia
}

\author{
Roberta Garbo, Andrea Mangiatordi, Silvia Negri \\ University of Milano Bicocca, Italy
}

\begin{abstract}
Note taking is a critical activity for success in higher education. In an inclusive perspective, it is important to make sure that all students, especially those with Learning Disabilities, can count on effective tools for carrying out this particular task. This preliminary study aimed at preparing the field for a larger study on a guided note taking system by investigating common behaviours and dynamics. Pen and paper note taking activity was observed on two selected subjects, one of whom with Dyslexia. After that, the same students were observed while using a note taking software allowing them to manipulate the content of the teacher's slides in order to create their own notes. Data from the class group context was also collected, in the form of questionnaires about the effectiveness of the lectures and about selfevaluation of performance. Information obtained from the two sources conflicted: students acting similarly had significantly different perceptions of their own effectiveness, or of the overall lecture effectiveness. This helped in shedding light on common misconceptions about note taking styles and led to the definition of a better testing procedure for the upcoming study.
\end{abstract}

\section{Introduction}

The 'Campus Tools for Students' (CATS) project [23], funded by the Italian Ministry of Education, University and Research (MIUR), aims at supporting students with hearing impairments and Learning Disabilities (LD), during classes, individual study, and fruition of administrative, ICT based services.

During lectures and in their everyday campus lives, both groups of students are at risk of losing a great deal of information delivered by teachers, of facing difficulties at various levels both during lecturing time and when it comes to individual study, of meeting with significant barriers when accessing didactic and administrative information in general. Specific tools and approaches are therefore required to meet their needs, thereby promoting equal opportunities for inclusion and active participation in the different aspects and phases of the educational process.

Among the main goals of the CATS project we could thus mention: analysing the difficulties faced by students with LD or hearing impairments, during lectures and individual study; designing approaches and solutions to support them in both conditions, personalising software services and promoting higher levels of accessibility. The research team leading the project has chosen to involve cooperatively students and teachers in evaluating the devised solutions, from the point of view of methodology and didactic use, with the objective of maximising the subjective quality and the effectiveness of the teaching-andlearning experience for all the actors involved. In fact, as a wide range of studies and international experiences have shown in the last two decades, design for all in education often starts from a careful field analysis of the difficulties and resources people with specific needs experience in performing what we could consider daily students' activities, and universities under this respect are no exception to the rule.

On this basis, the CATS team is evaluating every single step of the project, with the main purpose of collecting data about the present needs of the students and on the quality of the services/facilities available in campuses. The cornerstone of the whole project has been identified in moving from a culture of dependency to an inclusion model, driven by needs and oriented towards participation for all.

In order to comply with this basic assumption, the evaluation process for all devised solutions will be conducted on two different levels: first, their impact on students' performance will be measured adopting well-organized, repeatable experimental settings; second, an in depth analysis of further qualitative data will be performed, with a particular focus on the social impact of the introduction of campus tools on the personal learning experiences of university students.

This paper is organized as follows: section 2 contains a review of the literature linking dyslexia, note taking and information processing; section 3 describes the preliminary study we present, its participants and the methods adopted; section 4 provides a discussion of the collected data, with alternative interpretations of results and reflections on how the subsequent study should be organized; section 5 draws conclusions and outlines future work. 


\section{State of the art}

As the purpose of this paper is to describe a preliminary study of computer assisted note taking for university students, information was collected from literature about the kind of competences required and implied by this activity, existing resources useful for improving the quality of notes and different cognitive styles. This preliminary study was centred around a student with Dyslexia, hence a definition of this particular disorder is necessary since the beginning.

According to the official definition provided by the World Health Organisation [13], Dyslexia can be defined as "a disorder manifested by difficulty in learning to read, despite conventional instruction, adequate intelligence and sociocultural opportunity. It is dependent upon fundamental cognitive disabilities which are frequently of constitutional origin". Dyslexia can be recognized by the application of standardized tests, measuring reading achievement. Reading accuracy and speed of people with Dyslexia fall substantially below the expected values, given the individual's chronological age, measured intelligence, and age appropriate education Error! Reference source not found.

Many other definitions exist, from a multiplicity of authors: there is wide agreement on the fact that Dyslexia can only be diagnosed in absence of other problems in cognitive abilities (see [24] and Error! Reference source not found.).

Note taking is a critical activity for Dyslexic students, together with organization of essays and expressing ideas in writing [20]. Also, transcription fluency and reading comprehension are the best predictors of lecture notes, which in turn predict success in essays and memory tests [21]. There is evidence that students with dyslexia have more problems in selecting main ideas and in test taking strategies: they are often trained in the use of study aids and time management strategies in comparison to students without dyslexia [15]. They also perform poorly on tests and recall measures, even after recording lecture notes [4]. Moreover, research on university students with dyslexia also showed that they are more likely to report a deep approach to learning in comparison to those without dyslexia [15].

\subsection{Note taking techniques and software}

A variety of note taking techniques exist, sometimes with specific software to support them. As pointed out by [3], research in the field of note taking strategies for mild disabilities demonstrated the effectiveness of two main methods, called 'guided notes' and 'strategic note-taking'. The former method consists in providing students with outlines of the contents being explained, so that they can build their notes starting from a sort of preexistent 'skeleton' [17]. The latter method is based on organizing the note taking activity in steps, helping students focus their attention on teacher cues and vocabulary in the lecture; it also favours clustering similar lecture ideas and summarizing lecture points ([3], [5]).

During note taking it is common to use abbreviations, arrows, boxes and other graphical elements, but students can develop their own personal solutions mixing software and specific devices, such as OCR or portable recorders [29]. Research demonstrated that previous instruction can effectively improve the performance of students in lecture note taking [31].

Academic literature provides a huge amount of information on note taking software solutions: many of them have pen-based interfaces, as StuPad [28], Classroom Presenter [1], Souvenir [25] and NoteTaker [30]; some, as NoteLook [6] allow the integration of notes and digital video; NotePals [7] provides a way for sharing notes.

In recent times the diffusion of the Web contributed in augmenting the amount of information that every person is exposed to on a daily basis. This strengthened the need for note taking systems, not only for lectures. Tools like Evernote [12] or Diigo [10], among many others, are effective instruments for those who need to keep and organize information. A very popular software package as Microsoft Office [19] includes a tool specifically designed to facilitate note taking, called OneNote. However, choosing the right note taking system is not easy, mainly because people react to note taking devices very differently and current practices are not always well supported [14].

\subsection{Variability and cognitive styles}

According to the Universal Design for Learning (UDL) approach [22], curriculum design must be comprehensive of students' variability in learning.

Experimental evidence also demonstrated that the manipulation of instructional content can improve phonological working memory, also for students with Learning Disabilities [1]. Even though in this preliminary study participants were not provided with hands-on activities, an effort was made towards changing the typical note taking paradigm to allow a different kind of interaction with the instructional content provided by their teacher, in the vein of guided notes.

Research in the field of information processing ([3], [27]) highlighted the existence of different cognitive stiles. Such styles correspond to the strategies used to deal with a particular cognitive task. They represent preferences in the use of personal skills [26]. Cognitive styles are usually expressed as bipolar pairs, in which two elements 
represent extreme, mutually exclusive tendencies. Literature mainly agrees on the following pairs:

- global/analytic, as students can choose to start from a wide perspective on a topic, or to focus on details;

- systematic/intuitive, as a problem can be faced by step-by-step analysis or by formulating hypotheses and testing them;

- verbal/visual, as students can use textual summaries or visual representations of concepts;

- impulsive/reflexive, as the elaboration of contents can be fast but inaccurate or slow but accurate;

- dependent/independent from the context, as effectiveness in studying and working can be influenced from the context, or can happen freely in every situation;

- convergent/divergent, as learning can be guided by both logic and creativity.

As these styles apply to studying and, in general, to learning and problem solving, it is necessary to take them into account when choosing a note taking strategy, or when counselling about it a problematic student. The purpose of this work was not to find out the best note taking technology for students with Dyslexia, but to define a methodology for collecting data about students' perceptions of effectiveness related to their cognitive styles and their preferred strategies.

\section{The preliminary study}

The core of this work aimed at collecting useful contextual data in order to build the basis for a larger study. A mixed-methods approach was adopted in order to deepen the knowledge of this specific topic and to collect the largest possible amount of data from the observations. The following sections describe how participants were selected and how data collection was organized.

\subsection{Participants}

A form of reasoned sampling was adopted in order to select participants and their roles in the study: two of them received deeper attention, while the others acted as contextual reference.

At the beginning of the whole process a dyslexic student was identified, who from now on will be referred to as participant A; after that, one course was selected as a context for the observation. A second participant (who will be called $\mathrm{A}_{1}$ ) was chosen among a group of 12 volunteers attending that same course. The main criterion for this selection was having the highest number of traits in common with participant A, according to an initial questionnaire based on the above described cognitive styles, which will be discussed later.

The two participants were then two female students attending an Italian Literature course in their first year of an Education curriculum at the
University of Milano Bicocca, Italy. Both participated as volunteers, without the offer of a reward.

Other volunteers were also involved at a lesser degree: they will be referred to as 'the context group'. This group was composed of 5 students, who participated in the various phases of the study and in the data collection process. One of them was male, the others female. All of them were attending the same course in the same curriculum.

\subsection{Method}

The student selected as participant A was asked to refer the researchers to a teacher who used Power Point presentations on a regular basis as a teaching support. One teacher accepted the presence of the researchers during two of her classes. Her course, called "Italian Literature" was a mandatory one for students attending the first year of the Education curriculum.

The course group was visited before starting the observation session, during one of the classes of the last month of lessons. Students were asked to give their availability in participating in the preliminary study, explaining them that this would require answering an initial questionnaire. The email addresses of 12 students were then collected, and an invitation to an online survey was sent them the same day. The questionnaire included questions about:

- their competence level with ICT;

- their preferred methods for note-taking during lectures (pen and paper/with a computer/with audio recordings);

- their note-taking styles (schematic, discursive or mixed);

- their tendencies towards different cognitive styles (global/analytic, systematic/intuitive, verbal/visual, reflexive/impulsive, dependent/independent from the context, convergent/divergent);

- the teaching methodologies that better facilitated their learning efforts (frontal lecture, dialogic lecture, brainstorming, discussion, group work, simulations);

- the languages that better facilitated their learning efforts (oral, written, visual, multimedia and mathematic)

- the technologies that better facilitated their learning efforts (overhead projector, pc and projector, IWB)

- the degree of benefit they attributed to the use of PowerPoint supports during lectures.

The collected data was not anonymous: this allowed the use of the answers provided by participant A as a baseline. By a direct comparison of the other answer records with this baseline, participant $\mathrm{A}_{1}$ was selected, having a correspondence index of $82.8 \%$ (z-score 2,09) of answers (mean 57.5.\%, SD 12.1\%).

The other students who answered the questionnaire were also involved in the study, for 
two reasons: they allowed the collection of more "contextual data", and they allowed participant A to keep her anonymity. During the whole study, actually, no participant was informed that participants $\mathrm{A}$ and $\mathrm{A}_{1}$ would be subject to a more specific observation, neither that participant $A$ had Dyslexia.

The only divergences among the profiles of participants $A$ and $A_{1}$ related to: the use of the computer in note taking, used only by participant A; the tendency towards schematic note taking by participant $\mathrm{A}_{1}$; the prevalence of a reflexive style over systematic and impulsive behaviours for participant A. Answers to all other questions were identical. Surprisingly enough, participant A also answered she did not feel oral language to be a facilitator for her learning activity, while this is true for most dyslexic people.

The first observation took place one week after the selection of participants: the teacher was asked to prepare slides for her lecture, containing only text and images. Such slides mainly contained biographical notes about an author, book titles and images of book covers. The teacher was asked to act as she would have normally during the lecture. All participants (both $A$ and $A_{1}$, plus the others who volunteered for the questionnaire) were asked to sit in the first row, with participant $A$ at one end of the row and participant $A_{1}$ at the other. Two observers sat at their sides, with an observation grid in hand. The observation was conducted with a time sampling technique, using 5 minutes intervals. The total number of time slots for this phase was 16 .

The observation sheet featured a grid where each column referred to a time sample, while each row was associated to a different note-taking activity. Such list included: listening; writing keywords; writing discursive notes; writing schematic notes; writing what the teacher says (almost) verbatim; copying slides (almost) verbatim; interacting with the teacher; interacting with course mates. The last line allowed to mark the adoption of "other" activities or strategies.

At the end of the lecture a questionnaire was administered to all the participants, not only A and $A_{1}$. This survey included questions about the overall lesson course and, more specifically, about what students did during the lecture and while taking notes. Such data was useful for a comparison with the observation sheets. Two questions focused on the perceived efficacy of both the lesson per se and of the student's fruition of the event. Finally, a space was left for suggestions to the teacher about how to improve the efficacy of the lecture, and for reflections about how to strengthen students' efficacy. Additionally, participants $A$ and $A_{1}$ were asked to let the observers photocopy their notes.

The second observation session took place one week after the first. The main difference, due to the use of OneNote as a note-taking tool, was that it took place in a computer lab, where every student, also non-participants, had the possibility to access a computer. The total number of time slots for this phase was 16, as in the first lecture.

The teacher prepared another Power Point presentation, which was also transformed in a OneNote document before the lecture. This OneNote version was preloaded on the computers in the lab, in order to let participants use it as a guide for their notes.

Again, all the participants who showed up at class time were asked to sit in the front row, keeping the same disposition as in the previous observation. The observers used the same observation grid as before. At the end of the lecture the same final questionnaire was administered, adding only two questions: one about the usability of OneNote, the other about a comparison between the two lectures subject to observation. In this case it was easier to keep a copy of the notes of every participant, as they were saved as OneNote files and converted to PDF.

In analysing the data, particular attention was paid to:

- the answers given by participants $\mathrm{A}$ and $\mathrm{A}_{1}$ about the effectiveness of lectures in normal conditions and with the support of OneNote;

- the notes taken during the first and the second observation;

- the observation sheets resulting from the two sessions.

\section{Results}

The data collected during the two observation sessions included: the observation notes by two researchers, related to participants $A$ and $A_{1}$; the answers given to the final questionnaires by all participants; the static copies of the notes taken by participants $\mathrm{A}$ and $\mathrm{A}_{1}$.

The analysis will start from the observation notes, by highlighting the differences that exist between the performances of participants $A$ and $A_{1}$, both diachronically (each participant using different methods) and synchronically (both students using the same note taking method). Such notes will be integrated with the data coming from effectiveness questionnaires administered after each observation session. A second level of analysis will then consider the whole context group, relating only to their answers in the questionnaires.

\subsection{The two participants}

The most significative differences between the two observations of participant A, who had dyslexia, are:

- the time spent listening only, without taking notes, decreased drastically (15 counts during the first 
lecture, only 2 during the second). This could also be due to the natural differences between the contents of the two lectures: during the second observation the teacher introduced an individual sorting task, pausing frontal presentation;

- the notes taken with OneNote were less schematic than those taken using pen and paper (16 observations against 8 ), while the quantity of discursive notes observations increased (2 against 9);

- participant A started verbatim transcription of what the teacher was saying during the second lecture (no observations during the first session against 12);

- the time spent integrating and highlighting the notes decreased (13 observations against 5).

The other specific activities varied only slightly or not at all.

Also in the case of participant $A_{1}$, who is not dyslexic, there were some significant changes:

- schematic note-taking drastically decreased (it was observed 14 times during the first lecture, only once during the second);

- discursive note taking increased (2 observations against 11);

- she went over the notes already taken to apply small corrections only during the second lecture (11 observations of this activity);

- similarly, she deleted part of the notes taken during the same lesson, but only during the OneNote session (0 against 6 observations);

- interaction with classmates disappeared (4 observations in the first session, none in the second), but this could be also attributed to change in the environment which was introduced by the needs of the study.

There was no significant variation in the other specific activities.

It is then interesting to compare the data coming from the external observation with the answers given by participants to the questionnaires administered after the two lectures.

After the 'pen and paper' note taking session, both participants $A$ and $A_{1}$ declared they had taken schematic notes and attributed the maximum score to their perception of self-efficacy. Participant A did not answer the questions about the suggestions she would give to the teacher, while participant $A_{1}$ asked for a copy of the texts that were read during the lecture. Participant A also pointed out that she believed that her efficacy in taking notes could increase more if she had taken more schematic notes.

After the OneNote session, only participant A confirmed the high score attributed to her efficacy, while participant $A_{1}$ lowered the score by one point (on a 5 points scale). Participant A changed her style in note-taking, abandoning schematic notes in order to transcribe the teacher's speech verbatim, while participant $\mathrm{A}_{1}$ declared she kept taking schematic notes. Note that this answer conflicts with the data from external observations that was reported before, which suggests that the distinction between "schematic" and "discursive" is not really objective.

Both participants stated that OneNote did not present any usability issue, noticing that their note taking became more discursive. Again, the difference between schematic and discursive is problematic. Moreover, participant $A_{1}$, who registered an overall decrease in her efficacy, stated that OneNote facilitated her during the second lecture. Both appreciated the introduction of the visual language in their notes made possible by the software, considering it a facilitator for their learning. Participant A also highlighted the efficacy of the PowerPoint presentation.

It is also important to notice that software enriches and facilitates learning, but it is not a substitute for the teacher: both participants $\mathrm{A}$ and $\mathrm{A}_{1}$ attributed great part of the success of their learning to the use of frontal and dialogic lecture methods.

There are also interesting differences between the two participants in the two observation sessions. First of all, by comparing the grids, it was possible to determine how many times they happened to do exactly the same kind of action at the same time slot. Their correspondence was of $70 \%$ for pen and paper, but dropped to 38\% for OneNote.

During the first lecture it was clear that the main difference between them was the habit of reviewing notes whenever possible: this was a relevant activity for participant A (13 records) while participant $A_{1}$ did it fewer times (6 records).

More meaningful differences were observed during the second lecture:

- as reported earlier in this section, participant A reduced the amount of time spent only listening, while participant $A_{1}$ kept it at the same level as before;

- even if there is a doubt about the tendency to schematic or discursive notes for participant $A_{1}$, it is clear that participant A switched from discursive to schematic notes;

- participant A was the only one trying to transcribe what the teacher said verbatim;

- participant A put very little effort in reviewing her own notes, while her counterpart did it often (11 records), exactly reversing the tendency observed during the first lecture;

- the same happened with note deletion: participant A never deleted anything, while participant $A_{1}$ did it 6 times.

The clearer similarity observed between the two participants seems to be discursive note taking (9 observations for participant A, 11 for $A_{1}$ ). Actually, none of them declared to have taken more discursive notes in the final questionnaire, which strengthens the idea that a better classification of note taking styles is required. 
Many convergences can be found between participants according to the data deriving from the two final questionnaires.

After the first lecture participants $\mathrm{A}$ and $\mathrm{A}_{1}$ agreed on the high efficacy of the lesson, relating to the contents that were presented. The perception of efficacy of the frontal and dialogic lesson was also high, together with the use of oral and visual languages. While both had a high perception of their self-efficacy in taking notes and following the lecture, participant $\mathrm{A}$ appreciated more the use of the projector, while participant $A_{1}$ attributed greater importance to written and multimedia resources.

There were convergent points also in the second lecture:

- the dialogic method, together with visual language, was considered more effective;

- both participants agreed that OneNote was usable and that it did not create a barrier to note taking;

- the level of satisfaction for the effectiveness in attending the second lecture was higher than the first;

- notes were generally more discursive.

Even though the above similarities exist, the differences in the answers increased after the second lecture:

- participant $\mathrm{A}_{1}$ stated that the overall effectiveness of the lecture had decreased, while participant A kept the score on the same level as before;

- only participant A appreciated the effectiveness of frontal lecture method, of the use of oral language and of the PowerPoint presentation;

- the main activities of the two participants differed: as said before, participant A declared she had tried to transcribe the teacher's words, while participant $A_{1}$ was focused on schematic notes;

- self efficacy perception decreased only for participant $\mathrm{A}_{1}$;

- participant A was the only one reflecting on how to improve her future performance.

Observing the three pages of notes taken by participant A using pen and paper it was possible to notice that she used a mixed discursive/schematic approach, writing more in the first page and adding bullets and arrows in the second and third. Using OneNote, the same student transcribed more words, adopting a more discursive approach.

Participant $A_{1}$ was more schematic on pen and paper, using short sentences, rich of abbreviations, located in different parts of the sheet and linked using arrows, while the OneNote Document she produced, which was quite synthetic too, had larger sentences and reported many words as the teacher had said them.

\subsection{The context group}

The context group was originally composed of 8 students, apart from the two participants. Only 6 of them actually showed up at the first observation session and took the questionnaire. The others were not taken into account for the analysis. This means that a total of 8 students (6 as context, plus participants $A$ and $A_{1}$ ) were actually involved and had the opportunity to participate as volunteers. All of them completed both the initial cognitive styles survey form and the questionnaire at the end of the first observation session. Of these 6, only 4 participated also in the second observation session and completed the related questionnaire.

Table 1 shows the degree of correspondence with participant A that each other student reached in the initial cognitive styles survey and in the two observation sessions. As stated before, participant $A_{1}$ was chosen for observation because she was the most similar to participant A in terms of cognitive styles and preferences. The main difference between them was supposedly the diagnosis of Dyslexia.

Table 1 - Degree of correspondence between the answers given by each participant and the baseline

\begin{tabular}{rrrr} 
& $\begin{array}{c}\text { Cognitive } \\
\text { Styles } \\
\text { Survey }\end{array}$ & $\begin{array}{c}\text { Observation } \\
\text { Session 1 }\end{array}$ & $\begin{array}{c}\text { Observation } \\
\text { Session 2 }\end{array}$ \\
\hline $\mathrm{A}$ & baseline & \multicolumn{1}{c}{ baseline } & \multicolumn{1}{c}{ baseline } \\
$\mathrm{A}_{1}$ & $82.76 \%$ & $76.67 \%$ & $78.57 \%$ \\
$\mathrm{~B}$ & $72.41 \%$ & $76.67 \%$ & $N A$ \\
$\mathrm{C}$ & $55.20 \%$ & $N A$ & $N A$ \\
$\mathrm{D}$ & $51.72 \%$ & $80.00 \%$ & $78.57 \%$ \\
$\mathrm{E}$ & $51.72 \%$ & $83.33 \%$ & $78.57 \%$ \\
$\mathrm{~F}$ & $51.72 \%$ & $N A$ & $N A$ \\
$\mathrm{G}$ & $51.72 \%$ & $90.00 \%$ & $N A$ \\
$\mathrm{H}$ & $44.83 \%$ & $N A$ & $N A$ \\
$\mathrm{I}$ & $55.20 \%$ & $76.67 \%$ & $73.81 \%$ \\
\hline mean & $\mathbf{5 7 . 4 8 \%}$ & $\mathbf{8 0 . 5 6 \%}$ & $\mathbf{7 7 . 3 8 \%}$ \\
sd & $\mathbf{1 2 . 0 7 \%}$ & $5.34 \%$ & $\mathbf{2 . 3 8 \%}$ \\
\hline $\mathbf{A}_{\mathbf{1}} \mathbf{z - s c o r e}$ & $\mathbf{2 . 0 9}$ & $\mathbf{- 0 . 7 2}$ & $\mathbf{0 . 5}$ \\
\hline
\end{tabular}

It is immediately visible that the student expressing the more divergent opinion on the effectiveness of the first lesson was participant $A_{1}$. As the number of participants is very small, it is not possible to assume any correlation between cognitive styles and note taking performance, but it is still interesting to notice that in this case the highest degree of correspondence in evaluating the first lecture was obtained exactly by the participants who had less in common under the cognitive styles point of view.

The second observation yielded an equally interesting result: answers flattened around the mean value of $77.38 \%$, with students having different cognitive styles scoring the same. This could probably be attributed to the general lack of training 
in taking notes using a computer, which in some way made all participants equal.

Looking at the actual answers, and not at the relationships between participants, global, systematic and verbal cognitive styles are those in which the participants tended to recognize themselves more (they were chosen by 8, 7 and 7 students respectively). Participant A was the only one not identifying herself as systematic, but she did not select the opposite 'intuitive' style, which probably means she felt balanced between the two. Other popular choices were 'visual', 'reflexive' and 'convergent', each chosen 6 times.

Using k-means clustering techniques it was possible to divide the whole group of respondents in two clusters, according to their preferences in cognitive styles. Participants $\mathrm{A}$ and $\mathrm{A}_{1}$ were assigned by the k-means algorithm to two different groups. It was then necessary to find out which variable was setting them apart. Using the Kruskal-Wallis $\mathrm{H}$ test on single styles grouped by cluster it was possible to determine which of them characterized better the two subgroups. The lowest $p$-values were totalized by 'reflexive' $(p<0.001)$ and 'impulsive' $(p<0.01)$ styles. As it has already been pointed out in the previous subsection, participants $A$ and $A_{1}$ were mainly different under the cognitive styles point of view exactly for this pair. The other difference was that participant A did not identify herself as systematic. This styles-based clustering will be kept as a method of analysis for the subsequent study.

\section{Conclusions}

This preliminary study aimed at preparing the field for a larger study on a guided note taking system by investigating common behaviours and dynamics. The results of this mixed data collection helped in pointing out that classifying lecture notes is highly subjective: using categories such as 'schematic' and 'discursive' proved too problematic and dependent on personal interpretations. They make it harder to compare data collected through observation and through questionnaires. In the next phase of the research it will be necessary to preventively describe different aspects of note taking, in order to be able to classify students' answers and to link note taking styles to instruments.

Moreover, in order to make data more objective and accurate, a more analytical set of questions will be needed for evaluating both lecture and self effectiveness.

Another critical point is the gap between observation and questionnaire data: as it was pointed out in section 4, the comparison of observation grids suggested that participants $\mathrm{A}$ and $\mathrm{A}_{1}$ had acted very similarly with pen and paper, while their performances with the computer had followed very different paths. Such differences were not reflected in the two post-lecture questionnaires, which probably means again that there is too much space for subjectivity when note taking is involved. Due to the very short duration of the study, it is also possible that this contradiction happened by chance, so further investigation of this dimension will be required, together with a revision of the questions about effectiveness.

In order to scale the study to the level of a larger experiment it could also be interesting to include data from observation of students who use their notes to actually prepare for an exam, also administering effectiveness surveys. This would allow to understand the degree of relationship between how content is presented by teachers, how students take notes and how effective studying and learning is, specifically for students with Dyslexia.

Finally, more data could be collected in order to study the relationship between cognitive styles and note taking styles: the final objective will not be to find out the best approach to note taking, but how to put students in the condition of taking the best notes they can, depending on their personal qualities and their context. The specific case of dyslexic students is interesting mainly because they can already count on a variety of assistive tools, but they also need proper strategies and sufficiently inclusive environments.

\section{Acknowledgements}

The authors would like to thank Doctor Francesca Caputo of the University of Milano Bicocca, Italy for her kind support.

\section{References}

[1] Anderson, R., Anderson, R., Davis, K. M., Linnell, N., Prince, C., \& Razmov, V. "Supporting active learning and example based instruction with classroom technology", Proceedings of the 38th SIGCSE Technical Symposium on Computer Science Education (Covington, Kentucky, USA, March 07-11, 2007), SIGCSE '07 ACM, New York, NY, 2007, pp. 69-73

[2] Berninger, V. W., Winn, W. D., Stock, P., Abbott, R. D., Eschen, K., Lin, S.-J. (Cindy), Garcia, N., et al., “Tier 3 specialized writing instruction for students with dyslexia”. Reading and Writing, 21(1-2), 2007, pp. 95-129.

[3] Boyle, J., "Note-Taking and Secondary Students with Learning Disabilities: Challenges and Solutions", Learning Disabilities Research \& Practice, 27(2), 2012, pp. 90-101.

[4] Boyle, J. R., "Note-taking skills of middle school students with and without learning disabilities", Journal of Learning Disabilities, 43(6), 2010, pp. 530-540. 
[5] Boyle, J. R., "Strategic note-taking for middle school students with learning disabilities in science classrooms", Learning Disability Quarterly, 33 (2), 2010, pp. 93-109.

[6] Chiu, P., Kapuskar, A., Reitmeier, S., \& Wilcox, L., "NoteLook: taking notes in meetings with digital video and ink", Proceedings of the seventh ACM international conference on Multimedia (Part 1), 1999, pp. 149-158.

[7] Davis, R. C., Landay, J. A., Chen, V., Huang, J., Lee, R. B., Li, F. C., et al., "NotePals: Lightweight note sharing by the group, for the group", Proceedings of the SIGCHI conference on Human factors in computing systems: the CHI is the limit, 1999, pp. 338-345.

[8] De Beni, R., Pazzaglia, F., Moln, A., Zamperlin, C., Psicologia cognitive dell'apprendimento, Erickson, Trento, 2003.

[9] Dehane, S., Le neurones de la lecture, Odile Jacob, Paris, 2007.

[10] Diigo Inc., 'Diigo v. 5.0 beta', http://diigo.com $(28 / 8 / 2012)$.

[11] American Psychiatric Association, Diagnostic and statistical manual of mental disorders (4th ed., text rev.), Washington, DC, 2000.

[12] Evernote Corporation, 'Evernote', http://evernote.com (28/8/2012).

[13] World Health Organization, International Statistical Classification of Diseases and Health Related Problems, Geneva, 1990.

[14] Kim, K., Turner, S. A., \& Pérez-Quiñones, M. A. "Requirements for electronic note taking systems: A field study of note taking in university classrooms", Education and Information Technologies, 14(3), 2009, pp. 255-283.

[15] Kirby, J. R., Silvestri, R., Allingham, B. H., \& Fave, C. B. L., "Learning Strategies and Study Approaches of Postsecondary Students With Dyslexia”, Journal of Learning Disabilities, 41(1), 2008, pp. 85-96.

[16] Lancaster, P., "Universal Design for Learning”, Colleagues, 3(1), Article 5, 2008.

[17] Lazarus, B.D., "Flexible skeletons: Guided notes for adolescents with mild disabilities", TEACHING Exceptional Children, 28, 1996, pp. 36-40.

[18] Li, H., \& Hamel, C. M., "Writing issues in college students with learning disabilities: A synthesis of the literature from 1990 to 2000”, Learning Disability Quarterly, 26(1), 2003, pp. 29-46.

[19] Microsoft Corporation, 'Microsoft Office', http://office.microsoft.com/ (28/8/2012).

[20] Mortimore, T., Crozier, W. R., "Dyslexia and difficulties with study skills in higher education”, Studies in Higher Education, 31(2), 2006, pp. 235-251.
[21] Peverly, S. T., Sumowski, J. F., "What Variables Predict Quality of Text Notes and are Text Notes Related to Performance on Different Types of Tests?”, Applied Cognitive Psychology, 26(1), 2012, pp. 104-117.

[22] Rose, D., Meyer, A., Teaching Every Student in the Digital Age: Universal Design for Learning, ASCD, Alexandria, VA, 2002.

[23] Sbattella, L., Tedesco, R., Quattrini Li, A., Genovese, E., Corradini, M., Guaraldi, G., Garbo, R., Mangiatordi, A., Negri, S., “The CATS Project”, Advanced Information Technology in Education, Springer, Berlin/Heidelberg, 2012, pp. 265-272.

[24] Shaywitz \& Shaywitz, 2003.

[25] Spoerri, A., "Souvenir: Flexible note-taking tool to pinpoint and share media in digital libraries", Proceedings of the second ACM/IEEE-CS joint conference on Digital libraries, 2002, p. 383.

[26] Stella, G., and Grandi, L., Come leggere la dislessia e I DSA, Giunti Scuola, Firenze, 2012.

[27] Sternberg, R.J., Thinking styles, Cambridge University Press, Cambridge, MA, 1995.

[28] Truong, K. N., Abowd, G. D., "StuPad: Integrating student notes with class lectures”, $\mathrm{CHI}$ '99 extended abstracts on Human factors in computing systems, 1999, pp. 208-209.

[29] Wald, M., Draffan, E. A., Seale, J., "Disabled Learners' Experiences of E-learning”, Journal of Educational Multimedia and Hypermedia, 18(3), 2009 pp341-361.

[30] Ward, N., Tatsukawa, H., “A tool for taking class notes”, International Journal of Human-Computer Studies, 59(6), 2003, pp. 959-981.

[31] Wu, Y., "Effects of Note-Taking Instruction and Note-Taking Languages on College EFL Students ' Listening Comprehension", New Horizons in Education, 58(1), 2010, pp.120-133. 